\title{
MODELLING FARADAY ROTATION IN GALAXIES
}

\author{
LINDY BRETT \\ Department of Astronomy \\ University of Manchester \\ Manchester \\ M13 9PL
}

\begin{abstract}
Multi-frequency radio polarization observations of external galaxies are now available. The resolution is such that we can sample many positions over the galaxy, and different scale sizes. Faraday rotation measures, initial polarization angles, and depolarization can be used as indicators of magnetic field morphology in the source. In order to interpret the variation of polarization characteristics with frequency, a numerical model of Faraday rotation in galaxies is being developed. Preliminary results show that a wide range of situations can be investigated with the model.
\end{abstract}

\section{The Model}

A numerical model of Faraday rotation in emitting regions is being developed, which will be a general tool for predicting the polarization of emission from magnetic fields in radio sources, but with particular reference to spiral galaxies. The model consists primarily of a three dimensional mesh describing the variation of several parameters over a synthesized emitting region. From this, the Fourier transform relation between the distribution of Faraday depths in the region and the polarization characteristics of the beam is used to calculate the variation of the polarization with wavelength.

When thermal electrons are mixed with the emitting plasma, the predicted degree and angle of polarization vary in a complex way with wavelength, although an overall pattern of depolarization with increasing wavelength is predicted and observed. The modelling procedure described permits closer inspection of the variation of polarization with wavelength that is predicted for given combinations of magnetic fields and relativistic and thermal electrons.

Preliminary tests on the simple case of a uniform field in a slab replicate the predictions of theoretical analysis, showing depolarization and repolarization. A wide range of models can be developed by this method, from portions of a galactic disk to quasar environments. Models of galactic disks are being developed and will be compared with observations. It is also of interest to determine the relative importance of varying the magnetic field, thermal electrons, or relativistic electrons, in specific situations.

This work is funded by the Science and Engineering Research Council of the U.K. and is undertaken in co-operation with the Max-Planck-Institut für Radioastronomie in Bonn. 